Original Article

\title{
Characteristics of Postural Muscle Activation Patterns Induced by Unexpected Surface Perturbations in Elite Ski Jumpers
}

\author{
Hiroki Mani, PT, MS ${ }^{1)}$, Tatsuya IzUmi, PT, MS ${ }^{1)}$, Tomoya Konishi, PT, MS ${ }^{1)}$, \\ Mina Samukawa, PT, PhD $^{2}$, Keizo Yamamoto, PhD $^{3)}$, Kazuhiko Watanabe, $\mathrm{PhD}^{4}$, \\ TADAYOSHI ASAKA, PT, $\mathrm{PhD}^{2)^{*}}$ \\ 1) Graduate School of Health Sciences, Hokkaido University, Japan \\ 2) Faculty of Health Sciences, Hokkaido University: N12-W5, Kita-ku, Sapporo 060-0826, Japan \\ 3) Department of Sport Education, Hokusho University, Japan \\ 4) Center for Collaborative Research and Community Cooperation, Hiroshima University, Japan
}

\begin{abstract}
Purpose] This study investigated the characteristics of postural control following postural disturbance in elite athletes. [Subjects] Ten elite ski jumpers and ten control subjects participated in this study. [Methods] Subjects were required to maintain balance without stepping following unexpected horizontal surface perturbation in a forward or backward direction. [Results] A lower and reproducible peak magnitude of the center of mass velocity was shown in the athlete group compared to the control group. Cross-correlation analyses showed longer time lags at the moment of peak correlation coefficient between trunk flexor and extensor muscle activities, and shorter time lags and higher correlations between ankle flexor and extensor muscle activities were shown in the athlete group than in the control group. [Conclusion] The elite ski jumpers showed superior balance performance following surface perturbations, more reciprocal patterns in agonist-antagonist pairs of proximal postural muscles, and more cocontraction patterns in distal postural muscles during automatic postural responses than control individuals. This strategy may be useful in sports requiring effective balance recovery in environments with a dynamically changing surface, as well as in rehabilitation.
\end{abstract}

Key words: Postural reactions, Ski jumpers, Muscle activation pattern

(This article was submitted Nov. 11, 2013, and was accepted Jan. 7, 2014)

\section{INTRODUCTION}

Production of an adequate and coordinated postural response to a postural disturbance induced by an unexpected dynamically changing environment is crucial for effective balance recovery and fall prevention in individuals of all ages. In previous studies of postural control following a postural disturbance during standing, normal or inferior balance ability in healthy young, elderly or disabled individuals has been investigated ${ }^{1-5)}$. In contrast, the characteristics of superior postural control shown by highly skilled athletes that require excellent balance recovery in challenging conditions remain largely unknown ${ }^{6,7)}$. Knowledge of superior postural strategies could contribute to motor learning for effective balance recovery and fall prevention in the field of sports science, as well as in rehabilitation.

In able-bodied individuals, automatic postural responses (APR) are activated to correct disturbances to the body's

*Corresponding Author. Tadayoshi Asaka (E-mail: ask-chu@ hs.hokudai.ac.jp)

(C2014 The Society of Physical Therapy Science. Published by IPEC Inc. This is an open-access article distributed under the terms of the Creative Commons Attribution Non-Commercial No Derivatives (by-ncnd) License $<$ http://creativecommons.org/licenses/by-nc-nd/3.0/>. center of mass $(\mathrm{COM})^{8}$. APR onset occurs approximately $70-120 \mathrm{~ms}$ after spinal reflexes $(30-50 \mathrm{~ms})$, and before voluntary reactions $(220-280 \mathrm{~ms})^{9,}{ }^{9}$. In healthy individuals, "ankle" and "hip" strategies are considered the most typically coordinated patterns for maintaining stability in forward or backward surface translations, and they are based on the characteristic latency of distal-proximal postural muscle activations and the different ranges of motion of the major joints of the lower extremities ${ }^{8,9)}$. On the other hand, the major characteristics of individuals with inferior balance ability have been identified as larger fluctuations in COM or center of pressure (COP), delayed electromyogram (EMG) onsets ${ }^{4}$ and co-contraction patterns ${ }^{2,3,5,11)}$ of APR at the lower limb joints and trunk.

In previous studies of balance ability, athletes have generally shown superior balance ability compared to control subjects $^{12)}$. Davlin ${ }^{13)}$ reported that elite athletes such as gymnasts, swimmers and soccer players show superior balance ability, based on smaller fluctuations of COP under dynamic floor surface conditions, compared to non-athletes. In contrast, Noe and Paillard ${ }^{14)}$ reported that national-level skiers displayed postural performance inferior to that of regular skiers under static and dynamic conditions without ski boots. That is, national-level skiers showed larger displacements and faster velocities of COP than regular ski- 
ers. Among the few studies of postural responses evoked by perturbation in athletes, Johnson and Woollacott ${ }^{15)}$ indicated that power-trained athletes responded with shorter muscle-contraction onset times and larger muscle response amplitudes than endurance-trained athletes during APR.

Two typical muscle activation patterns, co-contraction and reciprocal patterns, have been identified in feedforward or feedback postural responses following forward-backward perturbations ${ }^{10,16,17)}$. Co-contraction patterns may be viewed as a means of increasing the apparent stiffness of the postural joints and stabilizing the body. On the other hand, reciprocal patterns may be more efficient at influencing important performance variables such as the COM, but also less safe under poorly predictable external conditions ${ }^{10,32)}$. However, the co-contraction/reciprocal activation patterns during APR of elite athletes have not been investigated.

The purpose of this study was therefore to investigate the characteristics of superior postural control of elite ski jumpers following surface perturbations. Ski jumping places high demands on the ability of the athlete to control posture and movement. It is the fine postural balance demanded at each phase of ski jumping (the in-run, take-off, stabilization after take-off and stabilization after landing) that makes it so difficult. The athlete has to solve extremely difficult sensorimotor tasks in real time and even minor mistakes can prohibit good performance in any one of the crucial phas$\mathrm{es}^{18)}$. Focusing on COM motion and postural muscle activation patterns in agonist-antagonist pairs at major joints of the lower extremities and trunk during this postural response, we postulated that 1) slower and more reproducible COM velocity, and 2) stronger reciprocal muscle activation patterns would be observed following perturbations in elite ski jumpers than in control subjects.

\section{SUBJECTS AND METHODS}

Ten elite ski jumpers of national/international level and ten healthy adults, all without any known neurological or motor disorders, participated in this study (Table 1). All the athletes had been training for ski jumping for 8-16 years. No subjects in the control group had participated at a national or international level of competition in any sport, but they had experience of playing sports such as basketball, soccer or badminton for 3-5 years. All subjects were rightleg dominant, as determined by the foot used to kick a ball and to step up onto a platform. Each subject provided their written informed consent in conformity with the principles of the Declaration of Helsinki (1964) and our local ethics committee.

Unilateral kinematic data in the sagittal plane were collected using a 6-camera motion-capture system (Motion Analysis Corporation, USA). Markers were placed at the following locations: the mastoid process, acromion, thigh, lateral femoral condyle, lateral malleolus, second metatarsal head, lateral epicondyle of the humerus, and wrist joint ${ }^{1,}$ 19). As the hanging arm obscured the position of the greater trochanter, the marker for the thigh was placed one-third the way along the line between the lateral femoral condyle and the greater trochanter, to allow calculation of the vir-
Table 1. General characteristics of the participants

\begin{tabular}{lcc}
\hline & Athlete $(\mathrm{n}=10)$ & Control $(\mathrm{n}=10)$ \\
\hline Age $(\mathrm{yr})$ & $19.8 \pm 2.4$ & $21.7 \pm 1.1$ \\
Weight $(\mathrm{kg})$ & $55.3 \pm 4.9$ & $57.7 \pm 6.8$ \\
Height $(\mathrm{cm})$ & $167.0 \pm 7.5$ & $169.3 \pm 6.3$ \\
BMR $\left(\mathrm{kg} / \mathrm{m}^{2}\right)$ & $19.8 \pm 0.5$ & $20.6 \pm 1.4$ \\
Gender $(\mathrm{male} /$ female $)$ & $6 / 4$ & $6 / 4$ \\
\hline
\end{tabular}

Values represent mean $\pm \mathrm{SD}$

tual position of the greater trochanter ${ }^{20)}$. Anthropometric measurements including ratio of segment mass to total body mass and ratio of segment COM to segment length were made. Signals from motion capture were digitized at a sampling frequency of $200 \mathrm{~Hz}$ and filtered with a $20 \mathrm{-Hz}$ lowpass, fourth-order, zero-lag Butterworth filter. Disposable self-adhesive electrodes (Ambu, Denmark) were used to record unilateral surface EMGs of the following muscles: the tibialis anterior (TA), medial head of gastrocnemius (GM), rectus femoris (RF), biceps femoris (BF), rectus abdominis (RA), and erector spinae (ES). Electrodes were placed on the left side of the body over the center of the muscle bellies (GM, RF and BF), 2 finger-breadths below the tuberosity of the tibia (TA), at the level of the umbilicus (RA), and at the level of L4 (ES), with their center points $3 \mathrm{~cm}$ apart ${ }^{21)}$. In addition, a reference electrode was attached to the lateral aspect of the fibula. Prior to the placement of the electrodes, the skin area was cleaned with alcohol and abraded with preparation gel to reduce skin surface impedance. The EMG system comprised two transmitters, two receivers and two amplifiers (Nihon Kohden Corporation, JP). EMG signals were amplified $(\times 2000)$ and digitized at a sampling frequency of $1,000 \mathrm{~Hz}$ with 12-bit resolution, then rectified and filtered with a $50-\mathrm{Hz}$ low-pass, fourth-order, zero-lag Butterworth filter.

Postural sway was induced by horizontal surface perturbation of the customized movable platform. The perturbation was adjustable in terms of movement distance and velocity. Sagittal plane balance was perturbed at random intervals by the movable platform in a forward or backward direction. The platform accelerated to $39 \mathrm{~cm} / \mathrm{s}$ for large perturbations ( $13 \mathrm{~cm} / \mathrm{s}$ for small perturbations) in an $80 \mathrm{~ms}$ interval, and the velocity was kept constant for $150 \mathrm{~ms}$ for large perturbations (300 $\mathrm{ms}$ for small perturbations), after which it decelerated and stopped in the following $80 \mathrm{~ms}$. The platform moved $10 \mathrm{~cm}$ or $5 \mathrm{~cm}$ in each direction in large or small perturbations, respectively.

The subjects stood barefoot with their feet side-by-side about shoulder width apart, and with their arms hanging at the sides of the body. In each trial, movement of the platform was triggered by an experimenter at random intervals from 1 to $5 \mathrm{~s}$ after a beep generated by a computer. Each subject was required to maintain balance without stepping, and was given three practice trials for each task prior to data collection. Subjects repeated the four tasks ( 2 perturbations [large and small] $\times 2$ directions) with 15 trials in each task. Subjects were exposed to 60 disturbances, with the trials randomized to minimize the ability of subjects to preplan 
responses. Rest periods of at least $5 \mathrm{~min}$ were provided between every 20 trials, based on a pilot study and our previous experience ${ }^{1)}$. If the subject displayed problems with stepping, the trial was discarded and repeated.

All signals were processed off-line using MatLab software (MathWorks, Natick, USA). Markers were used to calculate the COM, and a marker was attached to the movable platform. The initiation of horizontal surface movement (time zero: $t_{0}$ ) was defined as the point of first shift of displacement in the forward-backward direction of the marker attached to the movable platform. The body was modeled as a bilaterally symmetrical seven-segment model composed of feet, shanks, thighs, trunk, humerus, forearm, and head. Definitions of segment length proportions and inertial characteristics were based on the anthropometric data reported by Winter ${ }^{22}$. Filtered co-ordinate data were then combined with the anthropometric data to obtain position co-ordinates for the whole-body COM according to Brown et $\mathrm{al}^{19)}$. A detailed description can be found in the Appendix 1. For each trial, COM displacement was referenced to the position of the movable platform at each instant in time, and values were determined relative to the pre-translation COM position. Peak COM velocity during perturbation was then computed to explore subjects' ability to reduce postural disturbance. To evaluate the repeatability of wave forms of COM velocity during perturbation across trials, the adjusted coefficient of multiple correlation $(\mathrm{CMC})^{23)}$ was computed for each task. A detailed description can be found in Appendix 2. In order to normalize the CMC data, the data was log-transformed into $\mathrm{z}$ scores by Fisher's transformation.

To explore time-dependent covariates, that is, the relative strength of co-contraction in the flexor and extensor muscle activities involving the major joints of the lower extremities and trunk, cross-correlation analyses were run separately for EMGs in agonist-antagonist pairs ${ }^{2,3)}$ over a time period from $70 \mathrm{~ms}$ to $220 \mathrm{~ms}$ after $t_{0}$ to include APR. For each trial, the peak magnitude of the correlation coefficient (C-peak) and the time lag of C-peak were computed. A higher C-peak and shorter time lag of C-peak are viewed as more simultaneous bursts of muscle activity in agonist and antagonist pairs, and we subsequently describe this as "co-contraction" $" 24)$. GM for ankle plantar flexion and TA for ankle dorsal extension were selected to estimate ankle co-contraction. RF for knee extensor and BF for knee flexor were selected to estimate knee co-contraction. RA and ES were used to compute co-contraction at the trunk.

All of these parameters were computed and averaged across 15 trials for each task. Two-way analysis of variance (ANOVA) with factors Group (Athlete and control) and Task ( 2 perturbations $\times 2$ directions) was performed on the following dependent variables: the absolute value of the peak magnitude of COM velocity, z score of CMC, $\mathrm{C}$-peak, and the time lag of C-peak between the agonistantagonist muscle pairs at the ankle and knee joints, and trunk. Post-hoc analysis was performed using Bonferroni's comparisons when a main effect or interaction was significant. The relationship between the peak of COM velocity and the time lag for the combinations of two groups and two perturbed directions with large or small perturbations was investigated using Pearson's correlation coefficient. Statistical significance was accepted for values of $p<0.05$.

\section{RESULTS}

Figure 1 shows the time profiles of COM velocity in the forward-backward directions of both large and small perturbations for a typical subject in each group. The data represent average values and standard deviations across 15 trials of each task. Forward perturbation of the platform caused backward body sway, inducing an anterior postural response to recover COM. Backward perturbation of the plate induced the opposite response. A positive value on the y axis represents COM velocity in the forward direction. In control subjects, fluctuations in the time-series for COM velocity were observed in each task, specifically during the end of the acceleration phases and the start of the deceleration phases of the COM velocity. There was a significant main effect of Group on the absolute value of the peak magnitude of COM velocity $\left(F_{[1,16]}=43.42, p<\right.$ $0.01)$. The peak COM velocity was slower for the athlete group than for the control group. The average values and standard deviations of peak COM velocity of the athlete group were $43.6 \pm 2.5 \mathrm{~cm} / \mathrm{s}$ in backward large perturbations (BL), $13.0 \pm 1.1 \mathrm{~cm} / \mathrm{s}$ in backward small perturbations (BS), $39.0 \pm 2.0 \mathrm{~cm} / \mathrm{s}$ in forward large perturbations (FL) and 13.0 $\pm 0.7 \mathrm{~cm} / \mathrm{s}$ in forward small perturbations (FS). The data for the control group were: $49.6 \pm 3.9 \mathrm{~cm} / \mathrm{s}$ in $\mathrm{BL}, 15.9 \pm$ $0.9 \mathrm{~cm} / \mathrm{s}$ in BS, $43.9 \pm 3.2 \mathrm{~cm} / \mathrm{s}$ in FL and $17.1 \pm 1.3 \mathrm{~cm} / \mathrm{s}$ in FS. In addition, a significant effect of Task was found $\left(\mathrm{F}_{[3}\right.$, $\left.{ }_{48]}=1350.68, \mathrm{p}<0.01\right)$. Post-hoc analysis confirmed there was a significantly larger magnitude of peak COM velocity in large perturbations than in small perturbations in each direction $(\mathrm{p}<0.01)$. No significant interaction was found $\left(\mathrm{F}_{[3,48]}=1.87, \mathrm{p}>0.1\right)$. Furthermore, there was a significant main effect of Group on the $\mathrm{z}$ score of CMC $\left(\mathrm{F}_{[1,16]}=50.19\right.$, $\mathrm{p}<0.01)$. The $\mathrm{z}$ score of CMC was greater for the athlete group than for the control group. In addition, a significant effect of Task was found $\left(\mathrm{F}_{[3,48]}=85.38, \mathrm{p}<0.01\right)$. Post-hoc analysis confirmed there was significantly larger magnitude of the $\mathrm{z}$ score of CMC in the backward direction than in the forward direction in large perturbations $(p<0.01)$. No significant interaction was found $\left(\mathrm{F}_{[3,48]}=1.51, \mathrm{p}>0.1\right)$.

Figure $2 \mathrm{~A}$ shows time profiles of EMG patterns for a typical subject in each group. Data represent average values across the last 15 trials of FL. Note that flexor and extensor muscles of the ankle joint (TA and GM) activate simultaneously in a co-contraction pattern in the athlete subject. In contrast, EMG activities of flexor and extensor muscles of the trunk (RA and ES) showed a reciprocal pattern in the athlete subject, while the muscles of the trunk activate simultaneously in the control subject. Examples of correlation coefficients computed from cross-correlations between the rectus abdominis and erector spinae muscles from $50 \mathrm{~ms}$ to $200 \mathrm{~ms}$ after $\mathrm{t}_{0}$ for a typical subject in each group are shown in Fig. 2B. The data represent average values across the last 15 trials of FL. Time lag is defined as the lag time at the moment of peak correlation (C-peak). 

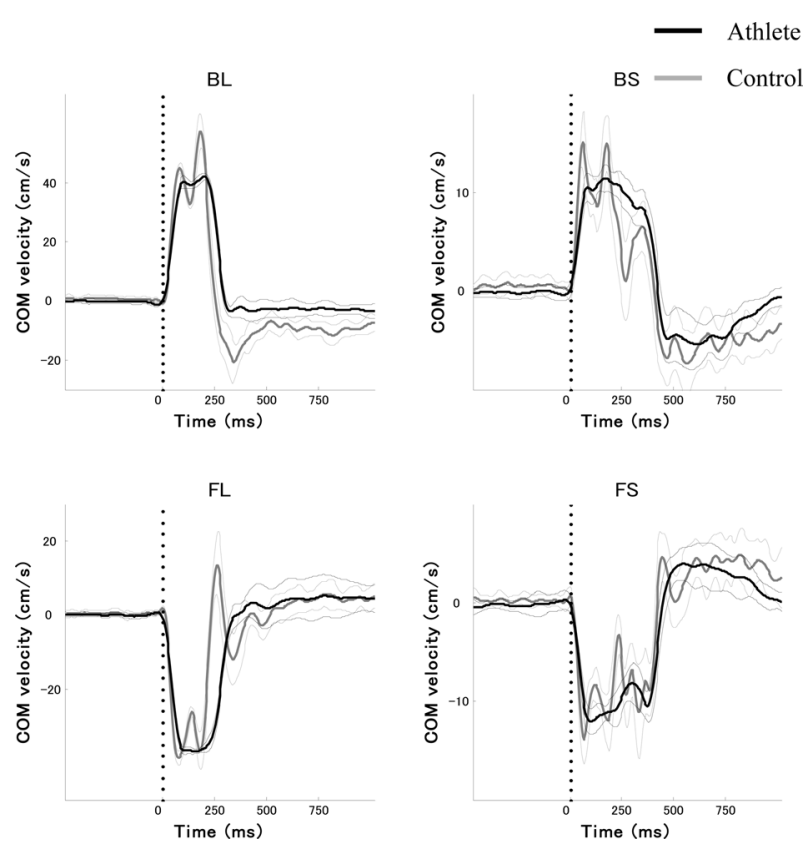

Fig. 1. Average time profiles with standard deviation of center of mass (COM) velocity across 15 trials in a typical subject from each group. Black and gray lines show subjects from the athlete and control groups, respectively. Vertical dotted lines show the initiation of perturbation $\left(t_{0}\right)$. BL, backward large perturbation; BS, backward small perturbation; FL, forward large perturbation; FS, forward small perturbation.

Table 2 shows average C-peak and time lag between flexor and extensor muscle activities in the ankle and knee joints, and trunk. Note that average time lags for the ankle were shorter in the athlete group than in the control group. There was a significant main effect of Group on the time lag between TA and GM $\left(\mathrm{F}_{[1,16]}=4.93, \mathrm{p}<0.05\right)$. In addition, a significant effect of Task was identified $\left(\mathrm{F}_{[3,48]}=18.14, \mathrm{p}<\right.$ $0.01)$. Post-hoc analysis confirmed there was a significantly longer time lag for the ankle in the backward direction than in the forward direction in large perturbations $(p<0.01)$. No significant interaction was found $\left(\mathrm{F}_{[3,48]}=1.45, \mathrm{p}>0.1\right)$. In contrast, average time lags for the trunk were longer in the athlete group than in the control group. There was a significant main effect of Group on the time lag between RA and ES $\left(\mathrm{F}_{[1,16]}=6.03, \mathrm{p}<0.05\right)$. No significant effect of Task or interaction was found $\left(\mathrm{F}_{[3,48]}=1.67,0.10\right.$, respectively, $\mathrm{p}>$ $0.1)$. Average time lags for the knee did not show significant effect of Group or interaction $\left(\mathrm{F}_{[1,16]}=2.54, \mathrm{~F}_{[3,48]}=0.39\right.$, respectively, $\mathrm{p}>0.1)$. In addition, a significant effect of Task was identified $\left(F_{[3,48]}=6.12, \mathrm{p}<0.01\right)$.

Average C-peaks of the ankle were larger in the athlete group than in the control group. There was a significant main effect of Group on C-peak of the ankle $\left(\mathrm{F}_{[1,16]}=6.14\right.$, $\mathrm{p}<0.05)$. No significant effect of Task or interaction was found $\left(\mathrm{F}_{[3,48]}=2.23,1.40\right.$, respectively, $\left.\mathrm{p}>0.1\right)$. Average $\mathrm{C}$ peaks of the knee did not show significant effect of Group or interaction $\left(\mathrm{F}_{[1,16]}=4.43, \mathrm{~F}_{[3,48]}=1.09\right.$, respectively, $\mathrm{p}$ $>0.05$ ). In addition, a significant effect of Task was identi-

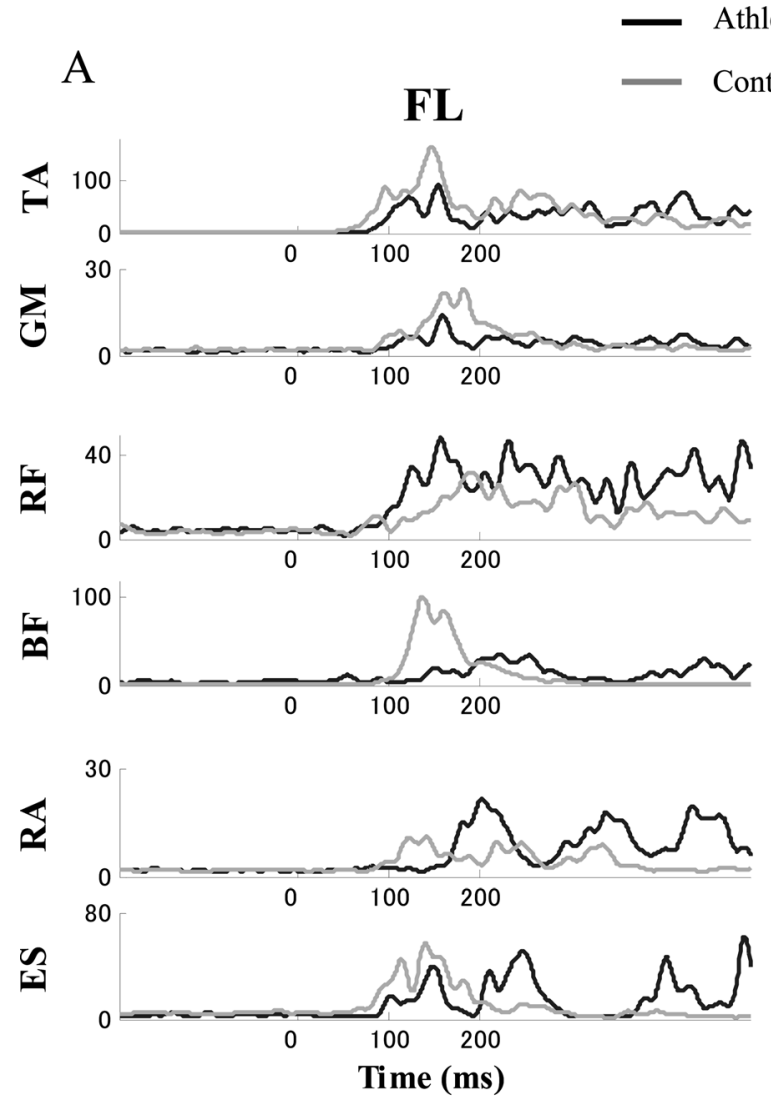

B

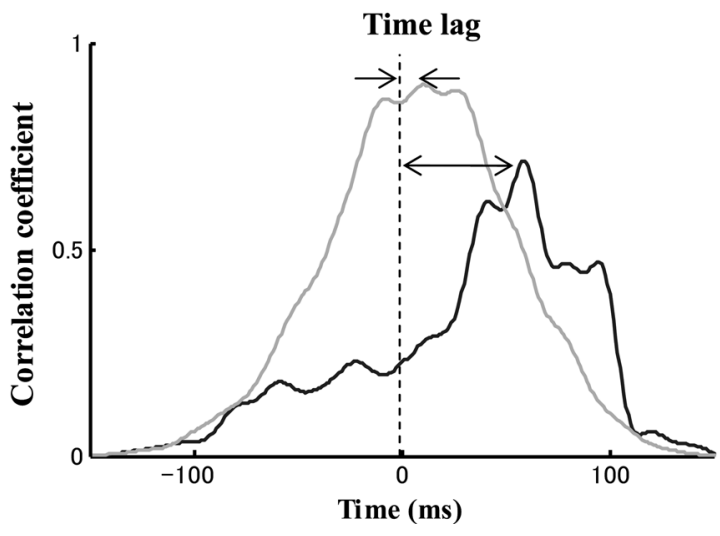

Fig. 2. A) Time profiles of EMG patterns for a typical subject in each group. B) An example of correlation coefficients computed from cross-correlations between the rectus abdominis and erector spinae muscles from $50 \mathrm{~ms}$ to $200 \mathrm{~ms}$ after $t_{0}$ for a typical subject in each group. Time lag is defined as the moment of peak correlation coefficient (C-peak). Data represent average values across the last 15 trials in forward large perturbation (FL). Black and gray lines represent subjects from the athlete and control groups, respectively. TA, tibialis anterior; GM, medial head of the gastrocnemius; RF, rectus femoris; BF, biceps femoris; RA, rectus abdominis; ES, erector spinae.

fied $\left(\mathrm{F}_{[3,48]}=6.45, \mathrm{p}<0.01\right)$. Average $\mathrm{C}$-peaks of the trunk did not show significant effect of Group, Task or interaction 
Table 2. Peak correlation coefficient (C-peak) and corresponding time lag between flexor and extensor muscle activities in the ankle and knee joints and trunk from $50 \mathrm{~ms}$ to $200 \mathrm{~ms}$ after the initiation of perturbation

\begin{tabular}{|c|c|c|c|c|c|c|c|c|}
\hline & \multicolumn{2}{|c|}{$\mathrm{BL}$} & \multicolumn{2}{|c|}{ BS } & \multicolumn{2}{|c|}{$\mathrm{FL}$} & \multicolumn{2}{|c|}{ FS } \\
\hline & Athlete & Control & Athlete & Control & Athlete & Control & Athlete & Control \\
\hline \multicolumn{9}{|c|}{ Ankle (TA \& GM) } \\
\hline C-peak & $0.87 \pm 0.02$ & $0.87 \pm 0.03$ & $0.86 \pm 0.04$ & $0.83 \pm 0.03$ & $0.90 \pm 0.04$ & $0.86 \pm 0.06$ & $0.89 \pm 0.03$ & $0.83 \pm 0.06 *$ \\
\hline time lag (ms) & $33.7 \pm 18.3$ & $34.2 \pm 12.1$ & $11.2 \pm 8.5$ & $25.0 \pm 12.0$ & $5.7 \pm 2.9$ & $17.0 \pm 14.0$ & $7.3 \pm 9.0$ & $17.9 \pm 16.2 *$ \\
\hline \multicolumn{9}{|l|}{ Knee (RF \& BF) } \\
\hline C-peak & $0.80 \pm 0.03$ & $0.84 \pm 0.03$ & $0.83 \pm 0.05$ & $0.85 \pm 0.04$ & $0.84 \pm 0.04$ & $0.86 \pm 0.03$ & $0.87 \pm 0.03$ & $0.87 \pm 0.03$ \\
\hline time lag (ms) & $26.1 \pm 9.4$ & $23.9 \pm 10.4$ & $16.4 \pm 12.8$ & $12.5 \pm 7.2$ & $22.4 \pm 11.3$ & $14.1 \pm 9.0$ & $14.4 \pm 9.8$ & $11.3 \pm 8.3$ \\
\hline \multicolumn{9}{|c|}{ Trunk (RA \& ES) } \\
\hline C-peak & $0.83 \pm 0.03$ & $0.84 \pm 0.03$ & $0.84 \pm 0.04$ & $0.83 \pm 0.05$ & $0.80 \pm 0.04$ & $0.83 \pm 0.04$ & $0.82 \pm 0.03$ & $0.82 \pm 0.04$ \\
\hline time lag (ms) & $40.3 \pm 14.7$ & $35.6 \pm 14.6$ & $30.5 \pm 17.0$ & $21.1 \pm 16.3$ & $33.3 \pm 14.5$ & $19.7 \pm 7.6$ & $30.7 \pm 21.2$ & $19.3 \pm 12.3 *$ \\
\hline
\end{tabular}

Values represent mean $\pm \mathrm{SD}$. TA, tibialis anterior; GM, medial head of the gastrocnemius; RF, rectus femoris; BF, biceps femoris; RA, rectus abdominis; ES, erector spinae. Abbreviations are the same as for Fig. $1 . * \mathrm{p}<0.05$.

$\left(\mathrm{F}_{[1,16]}=0.11, \mathrm{~F}_{[3,48]}=1.50, \mathrm{~F}_{[3,48]}=0.94\right.$, respectively, $\mathrm{p}$ $>0.1)$.

Peak values of COM velocity showed significant positive correlations with time lag of the ankle joint in combinations of the two groups and the two perturbation directions, with large or small perturbations $(r=0.45,0.40$, respectively, $p$ $<0.01)$. In fact, the shorter time lags of ankle joint were associated with slower COM velocity. In contrast, no significant correlation was seen between peak values of COM velocity and the time lag of the trunk in combinations of the two groups and the two perturbation directions, with large or small perturbations $(r=-0.22,-0.32$, respectively, $\mathrm{p}>0.05)$.

\section{DISCUSSION}

The results of this study reveal there were better performances in terms of COM velocity in the athlete group following not only large perturbations but also small perturbations, confirming our first hypothesis. That is, reduced peak magnitude and reproducible trajectories of COM velocity were shown in the athlete group compared to the control group (Figs. 1, 2). The results of the current study indicate elite skiers have superior ability to recover their posture, a result which seems to contradict Noe and Paillard's study cited in the Introduction. In their study, a platform with a movable pivot and only 1 degree of freedom of movement was used to analyze dynamic balance, and skiers were instructed to maintain the platform as horizontal as possible for $25.6 \mathrm{~s}$. Dynamic balance according to the methods of their study would thus mainly involve ankle strategy ${ }^{8}$, not whole-body postural recovery, and would require feedforward control as well as feedback control. However, superior postural control in elite athletes may be more apparent in postural conditions specific to the activity, such as wearing ski boots and standing with the knees flexed. In the current study, testing was performed with subjects standing barefoot in an upright position, because of the need to detect clear APR with low background muscle activity, in an imitation of previous studies ${ }^{4,8)}$.

Longer time lags of C-peak between trunk flexor and ex- tensor muscle activities were observed in the athlete group. Conversely, shorter time lags and higher correlations (Cpeak) between ankle flexor and extensor muscle activities were observed in the athlete group (Table 2). The short time lag and high correlation between agonist-antagonist muscle activities arise from the fact that the slite skiers showed a pattern of approximately simultaneous contraction (co-

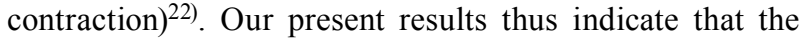
flexion-extension muscles of the trunk in the athlete group showed a more reciprocal pattern of activation than the control group, supporting our second hypothesis. In particular, large fluctuations in COM velocity at the end of acceleration and at the start of the deceleration phase of COM velocity were found in the control group (Fig. 1). In contrast, smooth trajectories of COM velocity roughly in accordance with the velocity of the movable platform were seen in the athlete group. Reciprocal patterns of proximal muscle activation may thus absorb inertial forces of rapid postural perturbations more easily than co-contraction patterns. We have shown that co-contraction patterns in feedforward postural responses occur more frequently under unstable conditions than under stable conditions, and changed to reciprocal patterns with practice ${ }^{17)}$. In addition, reciprocal patterns may be viewed as more efficient at controlling important performance variables such as $\mathrm{COM}^{10)}$. Taken together, we suggest that the higher reproducibility of COM velocities across repetitive trials in the athlete group is partly attributable to reciprocal patterns learned to motor learning under challenging conditions. Strong muscle strength ${ }^{25}$, instantaneous force ${ }^{26)}$, relevant proprioceptive organs ${ }^{27)}$, and prediction of postural control ${ }^{28)}$ in individual athletes would be other factors inhibiting fluctuations in COM velocity.

Interestingly, the results of this study indicate that the flexion-extension muscles at the ankle joint in the athlete group activated with more of a co-contraction pattern than in the control group. Furthermore, we report for the first time a relationship between peak COM velocities and time lags in agonist-antagonist muscle pairs at the ankle joint. In fact, the stronger the co-contraction pattern at the ankle joint was, the slower the peak COM velocities were. Increased co-contraction patterns have been observed under 
unstable conditions with a narrow base of support ${ }^{16,17)}$, with aging ${ }^{2,33)}$ and accompanying increased postural threat ${ }^{29)}$. We therefore suggest that elite ski jumpers reduce the rapid COM velocities through stiffness of the ankle joints, as evidenced by the co-contraction pattern at the ankle joints. This strategy of fixing distal joints rather than proximal joints following surface perturbations may illustrate a long-term effect of repetitive wearing of ski boots ${ }^{14)}$. This is because stiff ski boots restrict ankle joint motion ${ }^{30)}$, and restriction of ankle movement is known to exert significant effects on postural control ${ }^{31)}$.

Stiff ankle joints in conjunction with reciprocal patterns of proximal muscles would be effective at keeping the same base of support under challenging conditions. On the other hand, a stiff whole body, including stiff ankle joints due to muscular hypertonicity such as spasticity or rigidity, would result in poor balance after perturbation, since COM would not be recovered without reciprocal patterns. However, we failed to find a significant relationship between the peak COM velocities and the reciprocal patterns of proximal muscles in this study. It is possible that coordinated postural control between co-contraction patterns of distal muscles and reciprocal patterns of proximal muscles may be effective for recovery of the COM after surface perturbation. In addition, the muscle activation patterns of the failed trials in this study caused by stepping strategy to maintain balance would be worthy of investigation for rehabilitation purposes.

We conclude that elite ski jumpers show 1) slower and more reproducible COM velocity following horizontal surface perturbations, and 2) more reciprocal patterns in agonist-antagonist pairs of proximal postural muscles and more co-contraction patterns in agonist-antagonist pairs of distal postural muscles during automatic postural responses than control individuals. This strategy may be useful for effective balance recovery in environments with a dynamically changing surface in sports, as well as in rehabilitation. Postural control of athletes without footwear, such as that of elite gymnasts, is worthy of investigation in a future study.

\section{ACKNOWLEDGEMENT}

This research was supported by a Grant-in-Aid for Scientific Research (C) (21500461) from the Japan Society for the Promotion of Science.

\section{REFERENCES}

1) Asaka T, Yahata K, Mani H, et al.: Modulations of muscle modes in automatic postural responses induced by external surface translations. J Mot Behav, 2011, 43: 165-172. [Medline] [CrossRef]

2) Asaka T, Wang Y: Effects of aging on feedforward postural synergies. J Hum Kinet, 2008, 20: 63-70. [CrossRef]

3) Asaka T, Wang Y: Feedforward postural muscle modes and multi-mode coordination in mild cerebellar ataxia. Exp Brain Res, 2011, 210: 153-163. [Medline] [CrossRef]

4) Lin SI, Woollacott MH: Postural muscle responses following changing balance threats in young, stable older, and unstable older adults. J Mot Behav, 2002, 34: 37-44. [Medline] [CrossRef]

5) Küng UM, Horlings CG, Honegger F, et al.: Postural instability in cerebellar ataxia: correlations of knee, arm and trunk movements to center of mass velocity. Neuroscience, 2009, 159: 390-404. [Medline] [CrossRef]
6) Era P, Konttinen N, Mehto P, et al.: Postural stability and skilled performance - a study on top-level and naive rifle shooters. J Biomech, 1996, 29: 301-306. [Medline] [CrossRef]

7) Paillard T, Costes-Salon C, Lafont C, et al.: Are there differences in postural regulation according to the level of competition in judoists? $\mathrm{Br} \mathrm{J}$ Sports Med, 2002, 36: 304-305. [Medline] [CrossRef]

8) Horak FB, Nashner LM: Central programming of postural movements: adaptation to altered support-surface configurations. J Neurophysiol, 1986, 55: 1369-1381. [Medline]

9) Horak FB, Macpherson JM: Postural orientation and equilibrium. In: Handbook of physiology Section 12, Exercise regulation and integration of multiple systems. New York: Oxford University Press, 1996.

10) Robert T, Latash ML: Time evolution of the organization of multi-muscle postural responses to sudden changes in the external force applied at the trunk level. Neurosci Lett, 2008, 438: 238-241. [Medline] [CrossRef]

11) Benjuya N, Melzer I, Kaplanski J: Aging-induced shifts from a reliance on sensory input to muscle cocontraction during balanced standing. J Gerontol A Biol Sci Med Sci, 2004, 59: 166-171. [Medline] [CrossRef]

12) Hrysomallis C: Balance ability and athletic performance. Sports Med, 2011, 41: 221-232. [Medline] [CrossRef]

13) Davlin CD: Dynamic balance in high level athletes. Percept Mot Skills 2004, 98: 1171-1176. [Medline]

14) Noé F, Paillard T: Is postural control affected by expertise in alpine skiing? Br J Sports Med, 2005, 39: 835-837. [Medline] [CrossRef]

15) Johnson TK, Woollacott MH: Neuromuscular responses to platform perturbations in power- versus endurance-trained athletes. Percept Mot Skills, 2011, 112: 3-20. [Medline] [CrossRef]

16) Krishnamoorthy V, Latash ML, Scholz JP, et al.: Muscle modes during shifts of the center of pressure by standing persons: effect of instability and additional support. Exp Brain Res, 2004, 157: 18-31. [Medline] [CrossRef]

17) Asaka T, Wang Y, Fukushima J, et al.: Learning effects on muscle modes and multi-mode postural synergies. Exp Brain Res, 2008, 184: 323-338. [Medline] [CrossRef]

18) Müller W: Determinants of ski-jump performance and implications for health, safety and fairness. Sports Med, 2009, 39: 85-106. [Medline] [CrossRef]

19) Brown LA, Jensen JL, Korff T, et al.: The translating platform paradigm: perturbation displacement waveform alters the postural response. Gait Posture, 2001, 14: 256-263. [Medline] [CrossRef]

20) Milosavljevic S, Pal P, Bain D, et al.: Kinematic and temporal interactions of the lumbar spine and hip during trunk extension in healthy male subjects. Eur Spine J, 2008, 17: 122-128. [Medline] [CrossRef]

21) Basmajian JV, Blumenstein R: Electrode placement in EMG biofeedback. Baltimore: Williams \& Wilkins, 1980.

22) Winter DA: Biomechanics and motor control of human movements, 4th ed. New Jersey: John Wiley \& Sons, 2009.

23) Kadaba MP, Ramakrishnan HK, Wootten ME, et al.: Repeatability of kinematic, kinetic, and electromyographic data in normal adult gait. J Orthop Res, 1989, 7: 849-860. [Medline] [CrossRef]

24) Danna-Dos-Santos A, Degani AM, Latash ML: Anticipatory control of head posture. Clin Neurophysiol, 2007, 118: 1802-1814. [Medline] [CrossRef]

25) Neumayr G, Hoertnagl H, Pfister R, et al.: Physical and physiological factors associated with success in professional alpine skiing. Int J Sports Med, 2003, 24: 571-575. [Medline] [CrossRef]

26) Gruber M, Gollhofer A: Impact of sensorimotor training on the rate of force development and neural activation. Eur J Appl Physiol, 2004, 92: 98-105. [Medline] [CrossRef]

27) Paillard T, Noé F: Effect of expertise and visual contribution on postural control in soccer. Scand J Med Sci Sports, 2006, 16: 345-348. [Medline] [CrossRef]

28) McIlroy WE, Maki BE: Early activation of arm muscles follows external perturbation of upright stance. Neurosci Lett, 1995, 184: 177-180. [Medline] [CrossRef]

29) Carpenter MG, Frank JS, Silcher CP, et al.: The influence of postural threat on the control of upright stance. Exp Brain Res, 2001, 138: 210-218. [Medline] [CrossRef]

30) Schaff P, Hauser W: [Skin boot versus knee joint-a sports medicine, orthopedic and biomechanical problem]. Sportverletz Sportschaden, 1989, 3: 149-161. [Medline] [CrossRef]

31) Bennell KL, Goldie PA: The differential effects of external ankle support on postural control. J Orthop Sports Phys Ther, 1994, 20: 287-295. [Medline] [CrossRef]

32) Wang Y, Asaka T: Muscle synergies involved in shifts of the center of pressure while standing on a narrow support. Brain Res Bull, 2008, 76: 16-25. 
[Medline] [CrossRef]

33) Wang Y, Asaka T, Watanabe K: Multi-muscle synergies in elderly individuals: preparation to a step made under the self-paced and reaction time instructions. Exp Brain Res, 2013, 226: 463-472. [Medline] [CrossRef]

\section{APPENDIX}

1. $\mathrm{COM}=\mathrm{m} 1(\mathrm{x})+\mathrm{m} 2(\mathrm{x})+\mathrm{m} 3(\mathrm{x})+\mathrm{m} 4(\mathrm{x})+\mathrm{m} 5(\mathrm{x})+\mathrm{m} 6(\mathrm{x})+\mathrm{m} 7(\mathrm{x})$ $\mathrm{m} 1+\mathrm{m} 2+\mathrm{m} 3+\mathrm{m} 4+\mathrm{m} 5+\mathrm{m} 6+\mathrm{m} 7$

where $m=$ ratio of segment mass and $x=$ ratio of horizontal location of segment COM.

Ratios of the segment masses of the foot, shank, thigh, trunk, humerus, forearm, and head to total body mass were $0.015,0.047,0.100,0.467,0.028,0.016$ and 0.081 , respectively. Ratios of the proximal segment COM of the foot, shank, thigh, trunk, humerus, forearm, and head to the segment length were $0.500,0.433,0.433,0.500$, $0.436,0.430$ and 1.000 , respectively. COM of the head segment in the sagittal plane was defined at the ear canal ${ }^{22}$. Therefore, only one marker positioned on the surrounding ear canal, the mastoid process, was used to define the simplified head COM in the sagittal plane.
2. $\mathrm{CMC}$ is given by

$$
C M C=\sqrt{1-\frac{\sum_{j=1}^{N} \sum_{t=1}^{T}\left(Y_{j t}-\bar{Y}_{t}\right)^{2} / T(N-1)}{\sum_{j=1}^{N} \sum_{t=1}^{T}\left(Y_{j t}-\bar{Y}\right)^{2} /(N T-1)}}
$$

where $Y_{j t}$ is the $t$ th time point of the $j$ th trial,

$\bar{Y}_{t}$ is the average at time point $t$, where

$$
\bar{Y}_{t}=\frac{1}{N} \sum_{j=1}^{N} Y_{j t}
$$

and $\bar{Y}$ is the grand mean over time and is given by

$$
\bar{Y}=\frac{1}{N T} \sum_{j=1}^{N} \sum_{t=1}^{T} Y_{j t}
$$

where $N$ is 15 , and $T$ is $62(0-310 \mathrm{~ms})$ for large perturbation or $92(0-460 \mathrm{~ms})$ for small perturbation. 